\title{
Peritoneal adhesion prevention by a biodegradable hyaluronic acid-based hydrogel formed in situ through a cascade enzyme reaction initiated by contact with body fluid on tissue surfaces
}

Shinji Sakai*, Kohei Ueda, Masahito Taya

${ }^{\mathrm{a}}$ Division of Chemical Engineering, Department of Materials Science and Engineering,

Graduate School of Engineering Science, Osaka University, 1-3 Machikaneyama-cho, Toyonaka, Osaka 560-8531, Japan

*Corresponding author: Shinji Sakai

E-mail: sakai@cheng.es.osaka-u.ac.jp

Tel: +81-6-6850-6252; Fax: +81-6-6850-6254 


\begin{abstract}
Postsurgical peritoneal adhesion is a serious surgical complication. In situ hydrogel formation on the surface of tissues, which will develop adhesions, is a recent feasible approach to prevent peritoneal adhesion. Here, we report on-tissue surface formation of a hyaluronic acid-based hydrogel by administration of a pre-hydrogel aqueous solution. The hydrogelation was initiated by contact with body fluid containing glucose on tissue surfaces. During the hydrogelation, a hyaluronic acid derivative possessing phenolic hydroxyl moieties (HA-Ph) was cross-linked by a cascade reaction of glucose oxidase (GOx) and horseradish peroxidase (HRP). About 5 sec of hydrogelation was accomplished using a solution containing 1.5\% (w/v) HA-Ph, $5 \mathrm{U} / \mathrm{mL} \mathrm{HRP}$, and $2.5 \mathrm{U} / \mathrm{mL}$ GOx in $1 \mathrm{mg} / \mathrm{mL}$ glucose that is equivalent to the normal blood glucose concentration. The hydrogel was degradable by hyaluronidase and much softer than rat peritoneal sidewalls. We confirmed the efficiency of the hydrogel to prevent post-operative peritoneal adhesions by applying the solution containing $\mathrm{HA}-\mathrm{Ph}, \mathrm{GOx}$, and HRP to animals with bowel abrasion-abdominal sidewall defects. A significant reduction in the development of peritoneal adhesions was found compared with animals applied with phosphate-buffered saline or saline containing HA-Ph alone.
\end{abstract}

Keywords: Hyaluronic acid, Biodegradation, Hydrogel, Cascade enzyme reaction, Peritoneal adhesion prevention 


\section{Introduction}

Peritoneal adhesions are pathological bonds that usually occur between the abdominal wall, omentum, and loops of the bowel. They are mostly induced by surgery in the peritoneal cavity and frequently cause multiple serious complications such as chronic pelvic pain, small intestinal obstructions, and difficulties in re-operations [1, 2]. In the past three decades, various approaches have been examined to prevent these adhesions, including pharmacological and barrier-based approaches [1-3]. However, even now, peritoneal adhesions remain as a serious surgical complication. Recently, barrier-based approaches have attracted significant attention to prevent peritoneal adhesions. Polymer solutions [4, 5], preformed solid films and membranes [6, 7], and hydrogels [8-16] have been investigated for these approaches. Polymer solutions are useful to cover injured surfaces even with a complex geometry. However, persistent covering is greatly limited by the short residence time of the solution on the administrated site. For preformed solid films and membranes, it is difficult to cover injured surfaces with a complex geometry and extensive survey surface. A recent promising approach employs in situ cross-linkable hydrogels that can form hydrogels when applied in situ [8-14]. This strategy has advantages over approaches using polymer solutions and preformed solid films. The administered pre-hydrogel solution can cover injured surfaces with a complex geometry. Then, a hydrogel forms on the surface, resulting in a physical barrier to prevent direct contact with surfaces for a certain period of time. Injectable hydrogel systems can be classified into two types. One involves initiation of hydrogelation by mixing multiple cross-linkable precursor solutions immediately before application [8-11]. The other type initiates hydrogelation on the surface of the administered surface [12-14]. This system is simpler than the former type. One of these systems employing thermosensitive hydrogel has 
attracted attention because there is no need for additional factors such as ultraviolet light irradiation. The hydrogelation is induced by body temperature [14]. However, the relatively long time necessary for hydrogelation may be impractical. Therefore, there is still a need to develop an in situ gellable system that is triggered by the body.

Here, we describe a system employing a biodegradable hyaluronic acid (HA)-based hydrogel for peritoneal adhesion prevention, in which hydrogelation is initiated by contact with body fluids. The hydrogelation is based on co-enzymatic cross-linking mediated by a cascade reaction of glucose oxidase (GOx) and horseradish peroxidase (HRP) (Fig. 1a). HA and its derivatives have been previously used for adhesion prevention, including in situ gellable hydrogel systems because of their high biocompatibility and biodegradability [9-11]. In this study, we used the HA derivative possessing phenolic hydroxyl moieties (HA-Ph) developed by Kurisawa et al [17]. The feasibility of an HA-Ph hydrogel formed by HRP-catalyzed reaction has been investigated for various biomedical applications such as drug delivery and tissue engineering [18-20]. Hydrogelation of a HA-Ph aqueous solution containing HRP can be initiated by mixing with a solution containing $\mathrm{H}_{2} \mathrm{O}_{2}$, an electron donor of the HRP-catalyzed oxidative reaction, resulting in cross-linking between $\mathrm{Ph}$ moieties. GOx catalyzes the oxidation of glucose to $\mathrm{H}_{2} \mathrm{O}_{2}$ and glucono- $\delta$-lactone. Therefore, a solution containing glucose and GOx can be used as an alternative to a $\mathrm{H}_{2} \mathrm{O}_{2}$ solution. Glucose is a common component of body fluids. Thus, hydrogels can be formed by contacting an aqueous solution containing polymer with Ph moieties, HRP, and GOx with body fluids [21] (Fig. 1b). We examined the feasibility of a hydrogelation system consisting of a $\mathrm{HA}-\mathrm{Ph}$ aqueous solution containing HRP and GOx, in which hydrogelation is initiated by contact with body fluids for peritoneal adhesion prevention. 


\section{Materials and methods}

\subsection{Materials}

Sodium hyaluronate (MW:ca. 1,000 kDa) and GOx from Aspergillus niger (287 U/mg) were purchased from JNC (Tokyo, Japan) and Serva Electrophoresis GmbH (Heidelberg, Germany), respectively. Hyaluronidase from ovine testes (1100 U/mg), HRP (190 U/mg), N-hydroxy sulfosuccinimide (NHS), catalase $(10,000 \mathrm{U} / \mathrm{mg})$, and D-glucose were purchased from Wako Pure Chemical Industries (Osaka, Japan). Tyramine hydrochloride and 1-ethyl-3-(3-dimethylaminopropyl) carbodiimide (EDC) were purchased from Tokyo Chemical Industry (Tokyo, Japan) and Peptide Institute (Osaka, Japan), respectively. HA-Ph $\left(1.75 \times 10^{-4}\right.$ mol-Ph/g-HA) was synthesized by combining $\mathrm{HA}$ and tyramine via carbodiimide-mediated condensation of the carboxyl groups of HA and amino groups of tyramine using EDC and NHS according to a previous report [17].

\subsection{Gelation time}

The time necessary for hydrogelation of the HA-Ph solution through GOx and HRP-catalyzed reactions was evaluated by a previously reported method [22]. Briefly, $180 \mu \mathrm{L}$ phosphate-buffered saline (PBS, $\mathrm{pH}$ 7.4) containing HA-Ph, HRP, and GOx was added to a well of a 48-well plate and stirred with a magnetic bar. Subsequently, $20 \mu \mathrm{L}$ PBS containing glucose was added to the well with stirring. Swelling of the solution surface and hindrance of the magnetic stirring were considered to indicate hydrogelation. One of the concentrations of $\mathrm{HA}-\mathrm{Ph}, \mathrm{HRP}$, and GOx were altered to determine the effect of these parameters from the following condition: 1.5\% (w/v) HA-Ph, 5 U/mL HRP, and 2.5 U/mL GOx. The concentration of glucose in each well was fixed at $1 \mathrm{mg} / \mathrm{mL}$ that is equivalent to the normal blood glucose concentration. All solutions before mixing and the 48 -well plate were kept at $37^{\circ} \mathrm{C}$. The mean 
and standard deviations of triplicates were calculated.

\subsection{Biodegradability}

HA-Ph, HRP, and GOx were dissolved in PBS, and $1 \mathrm{~mL}$ of the solution was added to a cylindrical vessel (15 $\mathrm{mm}$ in diameter) with a dialysis membrane at the bottom. The vessel then was soaked in $12 \mathrm{~mL}$ PBS containing $1 \mathrm{mg} / \mathrm{mL}$ glucose for $12 \mathrm{~h}$ at $37^{\circ} \mathrm{C}$. After rising with PBS, the resultant hydrogel disk was soaked in PBS at $37^{\circ} \mathrm{C}$ for $2 \mathrm{~h}$. The hydrogel disks were then soaked in $13 \mathrm{~mL}$ PBS containing $50 \mathrm{U} / \mathrm{mL}$ hyaluronidase at $37^{\circ} \mathrm{C}$. Changes in the hydrogel weight were measured to evaluate degradation. The degree of degradation was calculated using the following formula: degradation $(\%)=\left\{\left(W_{0}-W_{\mathrm{t}}\right) / W_{0}\right\} \times 100$; where $W_{0}$ and $W_{\mathrm{t}}$ are the weights of hydrogel disks before and after soaking in PBS containing hyaluronidase, respectively. The mean and the standard deviations of triplicates were calculated.

\subsection{Mechanical properties}

Compression-repulsion stress profiles of HA-Ph hydrogel disks prepared under the conditions described in Section 2.3 were measured at room temperature using a tabletop materials tester (EZ-test, Shimadzu, Kyoto, Japan). The specimens were compressed at 5 $\mathrm{mm} / \mathrm{min}$ using a flat bottom probe. The means and standard deviations of triplicates at $50 \%$ compression were calculated. The compression-repulsion stress profile was also measured in the abdominal wall of male Sprague-Dawley rats after euthanasia by an overdose of pentobarbital (9 weeks old, Charles River Lab Japan, Kanagawa, Japan). All animal experiments and care were performed according to the 'Guide for the Care and Use of Laboratory Animals’ of Osaka University. 


\subsection{Cell adhesion in vitro}

Hydrogels prepared by 0.5 mL PBS containing 1.5\% (w/v) HA-Ph, 5 U/mL HRP, and 2.5 $\mathrm{U} / \mathrm{ml}$ GOx using procedure described in Section 2.3 were used to assess cell adhesion. The hydrogel disks were placed in 24-well plates covered with agarose gel. After several rinses with PBS and then with medium, 10T1/2 cells were seeded at $1 \times 10^{5} /$ well in $0.5 \mathrm{~mL}$ medium containing $10 \mathrm{U} / \mathrm{mL}$ catalase. After $24 \mathrm{~h}$ of incubation, cell adhesion on the hydrogels was observed under an optical microscope. The viabilities of the floating cells at $24 \mathrm{~h}$ after seeding were determined by a trypan blue exclusion test.

\subsection{Peritoneal adhesion prevention}

To analyze prevention of peritoneal adhesion, we used male CD1 (ICR) mice (10 weeks old, Charles River Lab Japan). Under pentobarbital anesthesia and carprofen analgesia, a $3 \mathrm{~cm}$ long midline incision was made. Abdominal adhesions were induced in 17 animals by establishing a $2 \times 2 \mathrm{~cm}$ defect on both the right and left lateral sidewalls by 80 strokes with a sterilized nylon toothbrush (Lion, Tokyo, Japan) until punctate bleeding occurred. The bowel was exteriorized and abraded by 40 times through application of digital pressure with a sterilized surgical gauge. Seven animals were randomly assigned to receive $1 \mathrm{~mL}$ PBS containing $1.5 \%(\mathrm{w} / \mathrm{v}) \mathrm{HA}-\mathrm{Ph}, 5 \mathrm{U} / \mathrm{mL} \mathrm{HRP}$, and $2.5 \mathrm{U} / \mathrm{mL}$ GOx by application onto the defects using a $1 \mathrm{~mL}$ plastic syringe. Five animals were randomly assigned to receive $1 \mathrm{~mL}$ PBS containing 1.5\% (w/v) HA-Ph but without GOx and HRP. The remaining five animals received $1 \mathrm{~mL}$ PBS. The abdominal cavities were closed by a double layer of sutures.

At 1 week after the treatments, five animals in each group (except for two animals treated with the solution containing $\mathrm{HA}-\mathrm{Ph}, \mathrm{GOx}$, and HRP) were euthanized by an overdose of pentobarbital. The remaining two animals were euthanized at 1 month after the procedure. Adhesions were scored for each abdominal side wall as follows [11]: $0=$ no adhesion, $1=$ 
tissue adherence that would separate with gravity, 2 = tissue adherence that would separate by blunt dissection, and $3=$ adhesion requiring sharp dissection. Tissues recovered from the necropsy were fixed in $10 \%$ formalin and then stained with hematoxylin and eosin for histological examination.

\subsection{Statistical analysis}

One-way ANOVA with Tukey’s post hoc analysis was used for comparisons between data sets. The values of $p<0.05$ were considered to indicate significance.

\section{Results and discussion}

\subsection{In vitro examinations}

The feasibility of HA-Ph hydrogels prepared by HRP-catalyzed reactions has been investigated in a variety of biomedical applications such as drug delivery and tissue engineering [18-20]. In this study, we applied HA-Ph hydrogels to barrier-based prevention of peritoneal adhesion, in which the hydrogel forms on the tissue surface by contact with body fluid. To initiate hydrogelation on tissue surfaces, we used GOx to supply $\mathrm{H}_{2} \mathrm{O}_{2}$ from the body fluid to the HRP-catalyzed reaction. In preliminary experiments, we confirmed the feasibility by the formation of HA-Ph hydrogels from an aqueous solution containing $\mathrm{HA}-\mathrm{Ph}, \mathrm{HRP}$, and GOx poured onto a dialysis membrane impregnated with fetal bovine serum (FBS) (Fig. 1c). Based on these results, we investigated the concentration-dependent effects of of HRP, GOx, and $\mathrm{HA}-\mathrm{Ph}$ on the time necessary for hydrogelation at the glucose concentration equivalent to the normal blood glucose concentration. The time required for hydrogelation decreased with increasing concentrations of HRP and GOx (Fig. 2). These trends are not specific to 
hydrogelation of a HA-Ph solution. Similar trends have been reported for various polymer-Ph solutions [21, 23]. In contrast, the effect of the $\mathrm{HA}-\mathrm{Ph}$ concentration was not significant compared with that of the enzymes. The important finding was a condition that enabled hydrogelation in around 5 sec. Such a short time would be fast enough for the formation of hydrogels at the administrated tissue surfaces before eluting off.

We next investigated the effect of HRP, GOx, and HA-Ph concentrations on the biodegradability of $\mathrm{HA}-\mathrm{Ph}$ hydrogels by hyaluronidase. Hydrogels prepared at lower concentrations of HRP, GOx, and HA-Ph degraded faster (Fig. 3). The HA-Ph hydrogels prepared by a solution containing 1.5\% (w/v) HA-Ph, 5 U/mL HRP, and 2.5 U/mL GOx were completely degraded after about $56 \mathrm{~h}$ of incubation at $37^{\circ} \mathrm{C}$. In contrast, hydrogels prepared at the lowest concentrations of HRP $\left(5 \times 10^{-3} \mathrm{U} / \mathrm{mL}\right)$ and GOx $\left(2.5 \times 10^{-3} \mathrm{U} / \mathrm{mL}\right)$ were completely degraded within $10 \mathrm{~h}$ of incubation. A possible explanation for the slow degradation of the HA-Ph hydrogels prepared at higher concentrations of HRP, GOx, and HA-Ph is a higher density of cross-links between Ph moieties. Penetration of hyaluronidase into hydrogels and degradation of individual $\mathrm{HA}-\mathrm{Ph}$ molecules by the enzyme would be hindered by a high cross-link density. Lee et at [24] studied the relationship between degradability by hyaluronidase and the microscopic structure and mechanical properties of HA-Ph hydrogels prepared by a mixture of $\mathrm{HA}-\mathrm{Ph}, \mathrm{HRP}$, and $\mathrm{H}_{2} \mathrm{O}_{2}$ without using GOx. They revealed that hydrogels with more cross-links are degraded more slowly than those with less cross-links [24]. The trend observed in this study is in agreement with these reported findings. The hydrogels prepared by higher concentrations of HRP, GOx, and HA-Ph showed a higher repulsion stress toward compression (Fig. 4) and were degraded more slowly by hyaluronidase (Fig. 3). Incidentally, it would be difficult to estimate the time necessary for disappearance of the HA-Ph hydrogels formed on tissue surfaces based on the results of this 
study. The physiological hyaluronidase concentration depends on the condition and location in the body [24, 25]. In addition, there are hyaluronidase inhibitors in serum [26]. An important finding in this study is the possibility of tailoring the degradation rate by changing HRP, GOx, and HA-Ph concentrations. The preparation conditions of HA-Ph hydrogels in practical clinical applications should be optimized after further studies that will be based on the findings obtained the current study.

Before the animal experiments employing a bowel abrasion-abdominal sidewall defect, we investigated the softness of the HA-Ph hydrogels and the abdominal sidewalls of animals. As shown in Fig. 5, the repulsion stress toward compression for the hardest HA-Ph hydrogel formed by the solution of $1.5 \%(\mathrm{w} / \mathrm{v}) \mathrm{HA}-\mathrm{Ph}, 5 \mathrm{U} / \mathrm{mL} \mathrm{HRP}$, and $2.5 \mathrm{U} / \mathrm{mL}$ GOx was much lower than that for the abdominal sidewalls. This result indicates a low risk of chafing to the tissue surfaces by contact with the hydrogel. In addition, we examined the adhesion of 10T1/2 fibroblastic cells to the hydrogel. Cells seeded on the HA-Ph hydrogel did not spread after 24 h of incubation (Fig. 6a). More than a half of the cells were washed off by gentle rinsing with PBS (Fig. 6b). In contrast, cells seeded on a cell culture dish attached and spread within $24 \mathrm{~h}$ of incubation (Fig. 6c). The non-spreading of the cells on HA-Ph hydrogel could have resulted from cell death, but we found $93.4 \%$ cell viability at $24 \mathrm{~h}$ of incubation on the hydrogels. These results demonstrate the low adhesiveness of the HA-Ph hydrogel. This feature would be useful to suppress fibrotic reactions to foreign bodies, which induce peritoneal adhesions. It has been reported that surfaces with low cell adhesiveness are effective for suppression of foreign body reactions to implanted bio-devices [27, 28].

\subsection{In vivo investigation}

According to the results of the in vitro experiments, we applied a solution of $1.5 \%(\mathrm{w} / \mathrm{v})$ 
HA-Ph, $5 \mathrm{U} / \mathrm{ml} \mathrm{HRP}$, and $2.5 \mathrm{U} / \mathrm{mL}$ GOx to mice with bowel abrasion-abdominal sidewall defects. As controls, PBS or a 1.5\% (w/v) HA-Ph solution were applied to five mice each with the same defects. At 1 week after the treatments, all mice treated with PBS or the HA-Ph solution (Fig. 7a) had developed adhesions on both sidewalls with an adhesion score of more than 2 (Table 1). In contrast, animals treated with the HA-Ph + HRP + GOx solution did not develop adhesions on six of 10 sidewalls (Fig.7 b). Adhesions with scores 1 and 2 were found on two sidewalls. Debris from the HA-Ph hydrogel was found in the abdominal cavities of all animals treated with the HA-Ph + HRP + GOx solution. Hydrogels were also found on some of the injured abdominal sidewall surfaces (Fig. 7b). The remaining hydrogel was also examined by histology of the sidewalls (Fig. 8b). Hydrogel adhesion at injury sites would be caused by hydrogel formation after penetration of the pre-hydrogel solution into wounds with a textured surface. Figure $8 \mathrm{~b}$ shows an example of hydrogels on the textured surface. No peritoneal adhesion was found in both two mice sacrificed at 1 month after treatment (Table 1). Continuous epithelialization was observed between the surface of the surrounding sidewalls which was assumed to be initially covered with hydrogels and the on-degrading remaining hydrogels (Fig. 8c). In addition, a smooth surface of muscle tissue contacting with the hydrogel indicated regeneration of the injured muscle tissues (Fig. 8c). The low but not non-adhesiveness of the HA-Ph hydrogel (Fig. 6b, c) would allow the epithelialization from surrounding tissues. Re-epithelization of an injury site after degradation of an in situ-formed hydrogel has been reported for hydrogels prepared by mixing hydrazide-modified HA with aldehyde-modified cellulose derivatives [10].

Another promising result was the existence of a smaller amount of inflammatory cells (neurophils and lymphocytes) around the hydrogels at 1 week (Fig. 8b) than in animals treated with PBS containing HA-Ph (Fig. 8a). In addition, no significant decrease in body 
weight was found compared with animals treated with PBS alone or the HA-Ph solution $(p>$ 0.05 by one-way ANOVA, Table 1). These results demonstrated that the HA-Ph hydrogel formed through the cascade enzyme reaction has a superior biocompatibility similarly to the HA-Ph hydrogels formed through a HRP-catalyzed reaction alone [17]. In addition, these results demonstrated the feasibility of applying the solution containing $\mathrm{HA}-\mathrm{Ph}, \mathrm{HRP}$, and $\mathrm{GOx}$ for peritoneal adhesion prevention.

A concern of the current system may be the use of GOx catalyzing the generation of $\mathrm{H}_{2} \mathrm{O}_{2}$, which is known to be cytotoxic [29], through consumption of glucose in body fluid even after hydrogel formation. In fact, when we used a solution without catalase, the viability of cells seeded onto HA-Ph hydrogels was around $30 \%$ after $24 \mathrm{~h}$ of incubation. The promising results of the in vivo experiments (Table 1, Figs. 7 and 8) would be obtained by a sufficient degradation rate of the generated $\mathrm{H}_{2} \mathrm{O}_{2}$ by catalase in vivo and/or the diffusion of GOx into the surrounding environment with subsequent clearance from the peritoneal cavity. Under the applied conditions in this study, we did not find an obvious adverse effect of GOx in the animal study. However, the concentration of GOx should be selected carefully when tailoring the characteristics of HA-Ph hydrogels, including the hydrogelation time. It was out of the scope of this study, however, other concerns may be the immunogenicities of HA-Ph, GOx, HRP and degradation product of HA-Ph. This issue should be carefully studied before clinical applications. To date, no adverse effects of $\mathrm{HA}-\mathrm{Ph}, \mathrm{HRP}$ and the degradation product of HA-Ph have been reported in 2- 8 weeks of animal studies [17, 18, 20, 30]. An additional concern may be the complexity of blending solutions before applying to patients. A possible solution is to supply as a commercial product after blending all the components based on optimized conditions. 


\section{Conclusions}

In this study, we investigated the feasibility of HA-Ph hydrogel formation initiated by contact with body fluid through GOx- and HRP-catalyzed reactions to prevent peritoneal adhesion. Using this method, the time necessary for hydrogelation, the biodegradability, and the mechanical properties of the resultant hydrogels can be modified by changing the concentrations of $\mathrm{HA}-\mathrm{Ph}, \mathrm{GOx}$, and HRP. The shortest gelation time at a normal concentration of blood glucose of $1 \mathrm{mg} / \mathrm{mL}$ was $5 \mathrm{sec}$. This hydrogel showed the highest resistance to degradation by hyaluronidase and the highest repulsion force toward compression but was much softer than the peritoneal sidewalls of rats. The utility of the proposed system was confirmed by peritoneal adhesion experiments using animals with bowel abrasion-abdominal sidewall defects. Six of the 10 sidewalls administrated the solution containing HA-Ph, GOx, and HRP did not develop peritoneal adhesions at 1 week after the treatment. In contrast, all sidewalls administrated PBS or the solution containing HA-Ph alone had developed adhesions. The promising result obtained for the proposed system primarily resulted from the prevention of adhesiogenesis by the hydrogel acted as a physical barrier on the injured site and the superior biocompatibility of the hydrogel resulting in no fibrous tissue formation. These results demonstrate the feasibility of administrating a solution containing HA-Ph, GOx, and HRP to tissue surfaces in the abdominal cavity to prevent post-operative peritoneal adhesion.

\section{Disclosure}

The authors indicate no potential conflicts of interest.

\section{Acknowledgement}


This work was financially supported by the Adaptable and Seamless Technology Transfer Program through target-driven R\&D, Japan Science and Technology Agency.

\section{References}

[1] Arung W, Meurisse M, Detry O. Pathophysiology and prevention of postoperative peritoneal adhesions. World J Gastroentero. 2011;17:4545-53.

[2] Brochhausen C, Schmitt V, Planck C, Rajab T, Hollemann D, Tapprich C, et al. Current strategies and future perspectives for intraperitoneal adhesion prevention. J Gastrointest Surg. 2012;16:1256-74.

[3] Yeo Y, Kohane D. Polymers in the prevention of peritoneal adhesions. Eur J Pharm Biopharm. 2008;68:57-66.

[4] Klink CD, Schickhaus P, Binnebösel M, Jockenhoevel S, Rosch R, Tolba R, et al. Influence of $4 \%$ icodextrin solution on peritoneal tissue response and adhesion formation. BMC Surg. 2013;13:34.

[5] Zheng Z, Zhang W, Sun W, Li X, Duan J, Cui J, et al. Influence of the carboxymethyl chitosan anti-adhesion solution on the TGF- $\beta 1$ in a postoperative peritoneal adhesion rat. $\mathbf{J}$ Mater Sci Mater Med. 2013;24:2549-59.

[6] Shahram E, Sadraie SH, Kaka G, Khoshmohabat H, Hosseinalipour M, Panahi F, et al. Evaluation of chitosan-gelatin films for use as postoperative adhesion barrier in rat cecum model. Int J Surg. 2013;11:1097-102.

[7] Pryor HI, O'Doherty E, Hart A, Owens G, Hoganson D, Vacanti JP, et al. Poly(glycerol sebacate) films prevent postoperative adhesions and allow laparoscopic placement. Surgery. 2009;146:490-7.

[8] Ito T, Yeo Y, Highley CB, Bellas E, Kohane DS. Dextran-based in situ cross-linked injectable hydrogels to prevent peritoneal adhesions. Biomaterials. 2007;28:3418-26.

[9] Li L, Wang N, Jin X, Deng R, Nie S, Sun L, et al. Biodegradable and injectable in situ cross-linking chitosan-hyaluronic acid based hydrogels for postoperative adhesion prevention. Biomaterials. 2014;35:3903-17.

[10] Ito T, Yeo Y, Highley CB, Bellas E, Benitez CA, Kohane DS. The prevention of 
peritoneal adhesions by in situ cross-linking hydrogels of hyaluronic acid and cellulose derivatives. Biomaterials. 2007;28:975-83.

[11] Takahashi A, Suzuki Y, Suhara T, Omichi K, Shimizu A, Hasegawa K, et al. In situ cross-linkable hydrogel of hyaluronan produced via copper-free click chemistry. Biomacromolecules. 2013;14:3581-8.

[12] Wei CZ, Hou CL, Gu QS, Jiang LX, Zhu B, Sheng AL. A thermosensitive chitosan-based hydrogel barrier for post-operative adhesions' prevention. Biomaterials. 2009;30:5534-40.

[13] Sawhney AS, Pathak CP, van Rensburg JJ, Dunn RC, Hubbell JA. Optimization of photopolymerized bioerodible hydrogel properties for adhesion prevention. J Biomed Mater Res. 1994;28:831-8.

[14] Zhang Z, Ni J, Chen L, Yu L, Xu J, Ding J. Biodegradable and thermoreversible PCLA-PEG-PCLA hydrogel as a barrier for prevention of post-operative adhesion. Biomaterials. 2011;32:4725-36.

[15] Lauder CI, Strickland A, Maddern GJ. Use of a modified chitosan-dextran gel to prevent peritoneal adhesions in a porcine hemicolectomy model. J Surg Res. 2012;176:448-54.

[16] Na SY, Oh SH, Song KS, Lee JH. Hyaluronic acid/mildly crosslinked alginate hydrogel as an injectable tissue adhesion barrier. J Mater Sci Mater Med. 2012;23:2303-13.

[17] Kurisawa M, Chung JE, Yang YY, Gao SJ, Uyama H. Injectable biodegradable hydrogels composed of hyaluronic acid-tyramine conjugates for drug delivery and tissue engineering. Chem Commun. 2005;34:4312-4.

[18] Lee F, Chung JE, Kurisawa M. An injectable hyaluronic acid-tyramine hydrogel system for protein delivery. J Control Release. 2009;134:186-93.

[19] Wang LS, Chung JE, Chan PP, Kurisawa M. Injectable biodegradable hydrogels with tunable mechanical properties for the stimulation of neurogenesic differentiation of human mesenchymal stem cells in 3D culture. Biomaterials. 2010;31:1148-57.

[20] Pek YS, Kurisawa M, Gao S, Chung JE, Ying JY. The development of a nanocrystalline apatite reinforced crosslinked hyaluronic acid-tyramine composite as an injectable bone cement. Biomaterials. 2009;30:822-8.

[21] Sakai S, Tsumura M, Inoue M, Koga Y, Fukano K, Taya M. Polyvinyl alcohol-based hydrogel dressing gellable on-wound via a co-enzymatic reaction triggered by glucose in the wound exudate. J Mater Chem B. 2013;1:5067-75.

[22] Sakai S, Kawakami K. Synthesis and characterization of both ionically and 
enzymatically crosslinkable alginate. Acta Biomater. 2007;3:495-501.

[23] Sakai S, Komatani K, Taya M. Glucose-triggered co-enzymatic hydrogelation of aqueous polymer solutions. RSC Adv. 2012;2:1502-7.

[24] Lee F, Chung JE, Kurisawa M. An injectable enzymatically crosslinked hyaluronic acid-tyramine hydrogel system with independent tuning of mechanical strength and gelation rate. Soft Matter. 2008;4:880-7.

[25] Fiszer-Szafarz B, Litynska A, Zou LM. Human hyaluronidases: electrophoretic multiple forms in somatic tissues and body fluids - Evidence for conserved hyaluronidase potential $N$-glycosylation sites in different mammalian species. J Biochem Biophys Methods. 2000;45:103-16.

[26] Kolliopoulos C, Bounias D, Bouga H, Kyriakopoulou D, Stavropoulos M, Vynios DH. Hyaluronidases and their inhibitors in the serum of colorectal carcinoma patients. J Pharm Biomed Anal. 2013;83:299-304.

[27] Ishiyama N, Moro T, Ohe T, Miura T, Ishihara K, Konno T, et al. Reduction of peritendinous adhesions by hydrogel containing biocompatible phospholipid polymer MPC for tendon repair. J Bone Joint Surg Am. 2011;93:142-9.

[28] Anderson JM, Rodriguez A, Chang DT. Foreign body reaction to biomaterials. Semin Immunol. 2008;20:86-100.

[29] Gulden M, Jess A, Kammann J, Maser E, Seibert H. Cytotoxic potency of $\mathrm{H}_{2} \mathrm{O}_{2}$ in cell cultures: Impact of cell concentration and exposure time. Free Rad Biol Med. 2010;49:1298-305.

[30] Wang LS, Lee F, Lim J, Du C, Wan AC, Lee SS, et al. Injectable biodegradable hydrogels composed of hyaluronic acid-tyramine conjugates for drug delivery and tissue engineering. Acta Biomater. 2014; 10:2539-50.

Table 1. Body weight changes and adhesion scores of injured sites* established on both the righ and left lateral sidewalls in peritoneal adhesion prevention experiments.

\begin{tabular}{lllll}
\hline & $\begin{array}{l}\text { PBS } \\
(1 \text { week })\end{array}$ & $\begin{array}{l}\text { HA-Ph solution } \\
(1 \text { week })\end{array}$ & $\begin{array}{l}\text { HA-Ph }+ \text { HRP }+ \\
\text { GOx (1 week) }\end{array}$ & $\begin{array}{l}\text { HA-Ph }+ \text { HRP }+ \\
\text { GOx (1 month) }\end{array}$ \\
\hline Body weight increase (\%) & $-2.1 \pm 5.9^{* *}$ & $-4.1 \pm 4.6^{* *}$ & $0.3 \pm 2.6^{* *}$ & $13.7 \pm 2.0$ \\
Score 0 & 0 & 0 & 6 & 4 \\
Score 1 & 0 & 0 & 2 & 0 \\
Score 2 & 2 & 0 & 2 & 0 \\
Score 3 & 8 & 10 & 0 & 0 \\
\hline
\end{tabular}

* $0=$ no adhesion, 1 = tissue adherence that would separate with gravity, 2 = tissue adherence that would separate 


\section{Figure Captions}

Fig. 1. a) Schematic of covering an abdominal tissue surface with a HA-Ph hydrogel by pouring a gellable solution containing $\mathrm{HA}-\mathrm{Ph}, \mathrm{HRP}$, and GOx onto the tissue surface. b) Scheme for HA-Ph hydrogel formation via GOx- and HRP-catalyzed reactions by consuming glucose in body fluid. c) Image of a pinched HA-Ph hydrogel formed by pouring a solution containing HA-Ph, HRP and GOx onto a dialysis membrane moistened with FBS.

Fig. 2. Dependence of hydrogelation time on the concentrations of HRP, GOx, and $\mathrm{HA}-\mathrm{Ph}$ in the pre-hydrogel aqueous solution. The gelation time was measured at $37^{\circ} \mathrm{C}$ by altering one of the concentrations of $1.5 \%$ (w/v) HA-Ph, $5 \mathrm{U} / \mathrm{mL}$ HRP, or $2.5 \mathrm{U} / \mathrm{mL}$ GOx. Data represent the means with standard deviations.

Fig. 3. Dependence of HA-Ph hydrogel degradation on the concentrations of HRP, GOx, and $\mathrm{HA}-\mathrm{Ph}$ in the pre-hydrogel aqueous solution. The degradation in an aqueous solution containing $50 \mathrm{U} / \mathrm{mL}$ hyaluronidase was measured at $37^{\circ} \mathrm{C}$ for the hydrogels formed by altering one of the concentrations of $1.5 \%$ (w/v) HA-Ph, $5 \mathrm{U} / \mathrm{mL}$ HRP, or $2.5 \mathrm{U} / \mathrm{mL}$ GOx. Data represent the means with standard deviations.

Fig. 4. Dependence of repulsion stress of $\mathrm{HA}-\mathrm{Ph}$ hydrogels at $50 \%$ compression on the concentrations of HRP, GOx, and $\mathrm{HA}-\mathrm{Ph}$ in the pre-hydrogel aqueous solution. The dependence was measured for the hydrogels formed by altering one of the concentrations of 1.5\% (w/v) HA-Ph, 5 U/mL HRP, or $2.5 \mathrm{U} / \mathrm{mL}$ GOx. Data represent the means with standard deviations. ${ }^{*} p<0.05$. 
Fig. 5. Compression-repulsion stress profiles of the abdominal sidewall and HA-Ph hydrogel formed by a solution containing 1.5\% (w/v) HA-Ph, 5 U/mL HRP, and 2.5 U/mL GOx. Data represent the means with standard deviations.

Fig. 6. Photographs of $10 \mathrm{~T} 1 / 2$ cells at $24 \mathrm{~h}$ after seeding on (a) a cell culture dish or (b, c) a HA-Ph hydrogel. (b) Before and (c) after rinsing with PBS. Bars: $100 \mu \mathrm{m}$.

Fig. 7. Abdominal sidewalls of mice treated with solution containing (a) $1.5 \%$ (w/v) $\mathrm{HA}-\mathrm{Ph}$ or (b, c) 1.5\% (w/v) HA-Ph, 5 U/mL HRP, and $2.5 \mathrm{U} / \mathrm{mL}$ GOx at (a, b) 1 week and (c) 1 month after treatment.

Fig. 8. Hematoxylin and eosin-stained sections of the abdominal sidewalls of mice treated with solution containing (a) 1.5\% (w/v) HA-Ph or (b, c) 1.5\% (w/v) HA-Ph, 5 U/mL HRP, and $2.5 \mathrm{U} / \mathrm{mL}$ GOx at (a, b) 1 week and (c) 1 month after treatment. Bars: $100 \mu \mathrm{m}$. 
a)

\section{$\mathrm{HA}-\mathrm{Ph}$ \\ + HRP solution \\ + GOx}

Body fluid

\section{Tissue surface}

b)

Glucose
in body fluid

$$
\mathrm{O}_{2} \quad \mathrm{H}_{2} \mathrm{O}_{2} \quad \mathrm{H}_{2} \mathrm{O}
$$

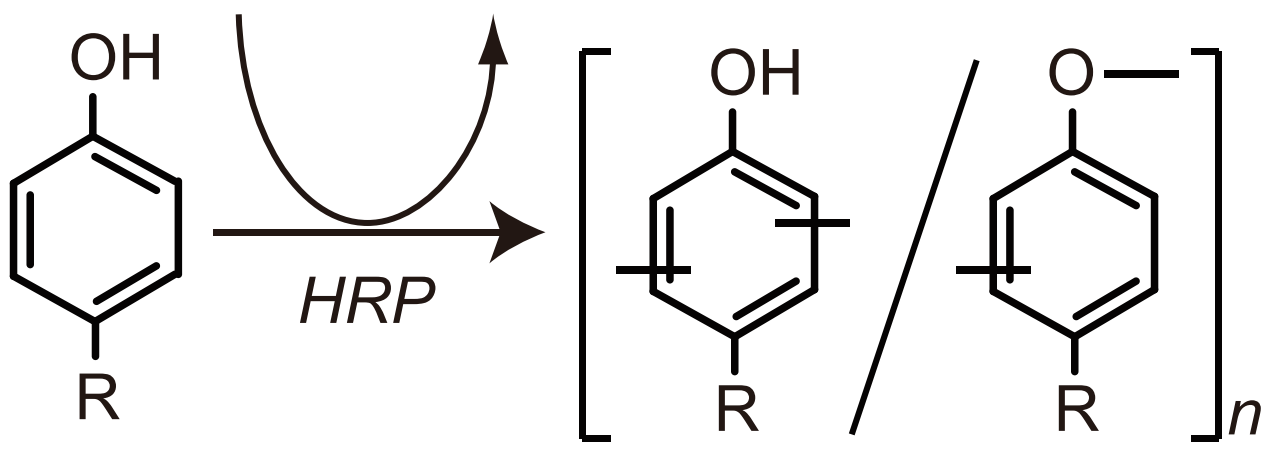

c)

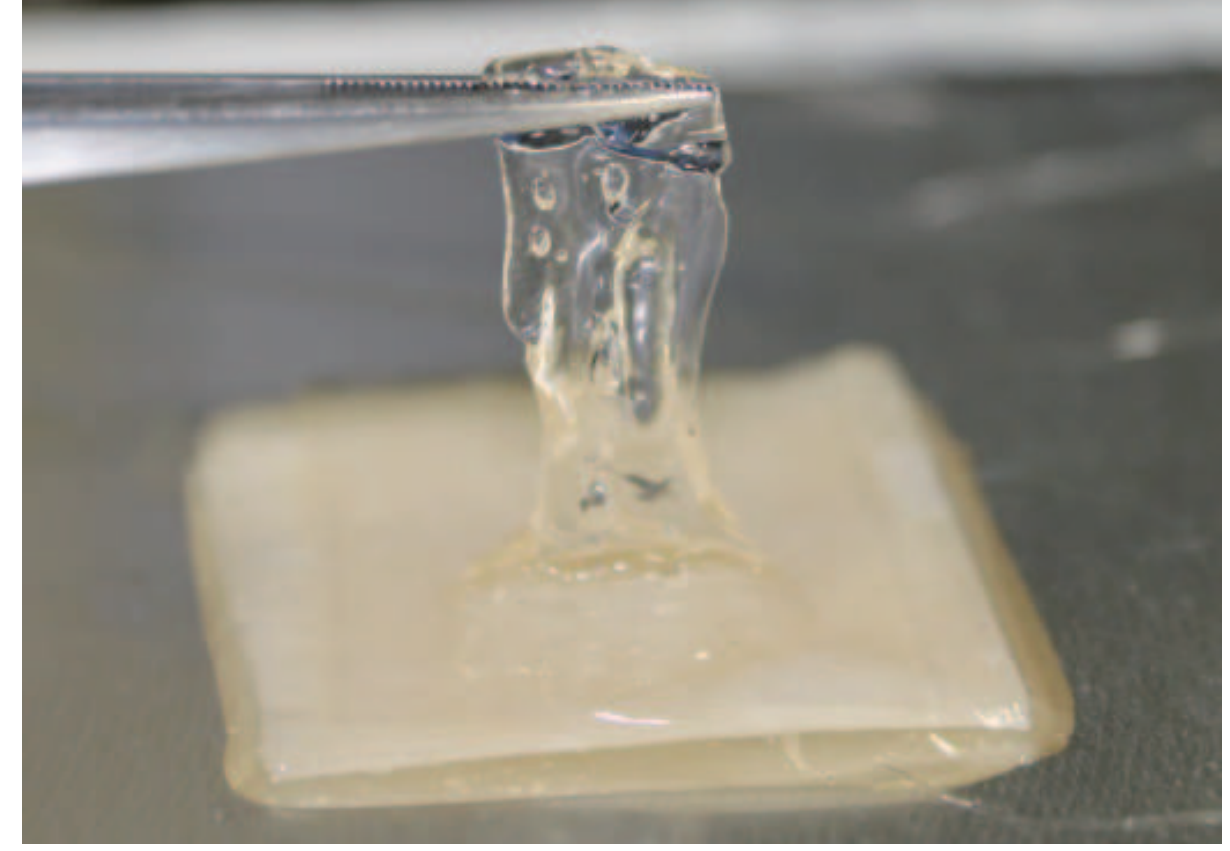


Figure 2

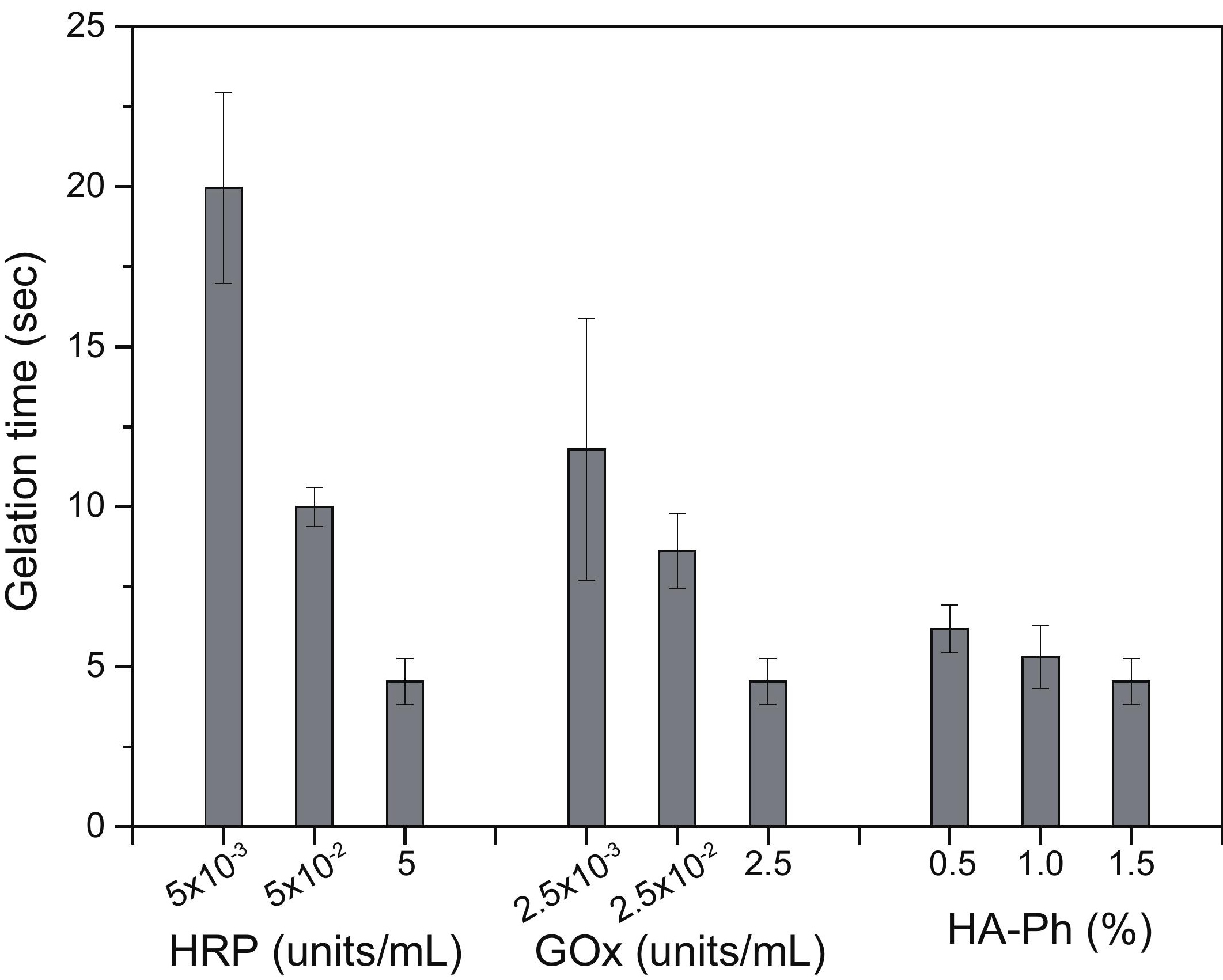




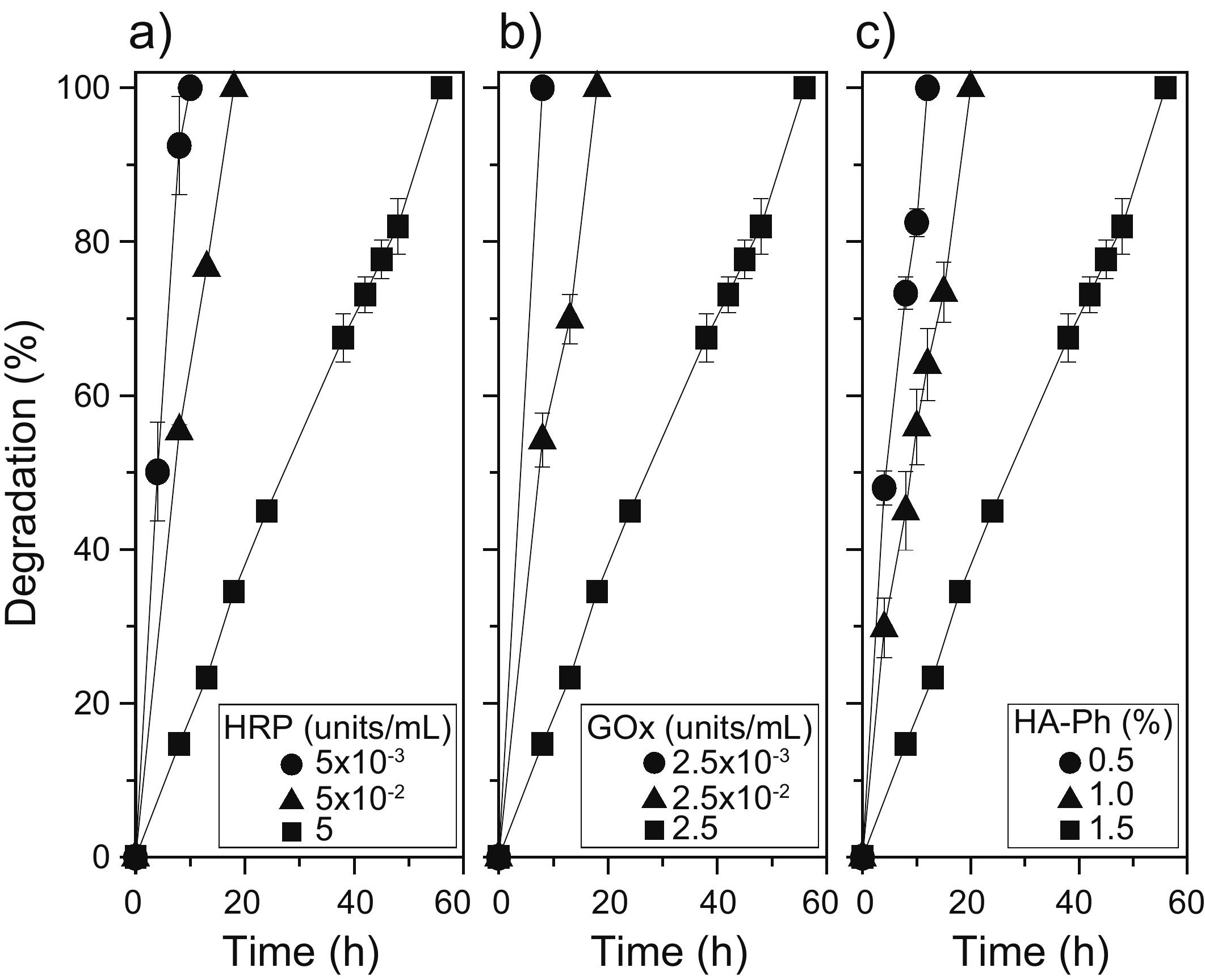




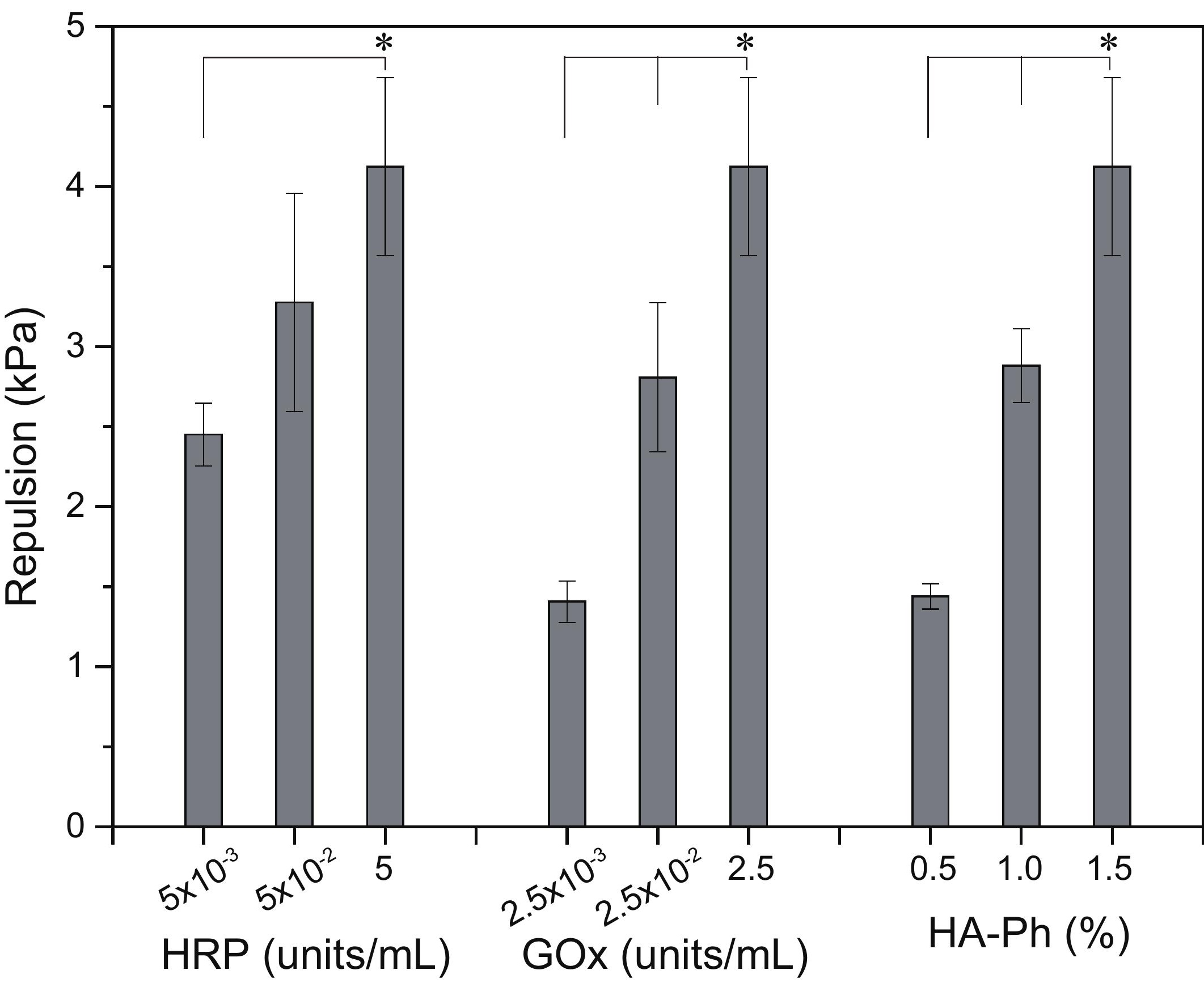




\section{$a$}

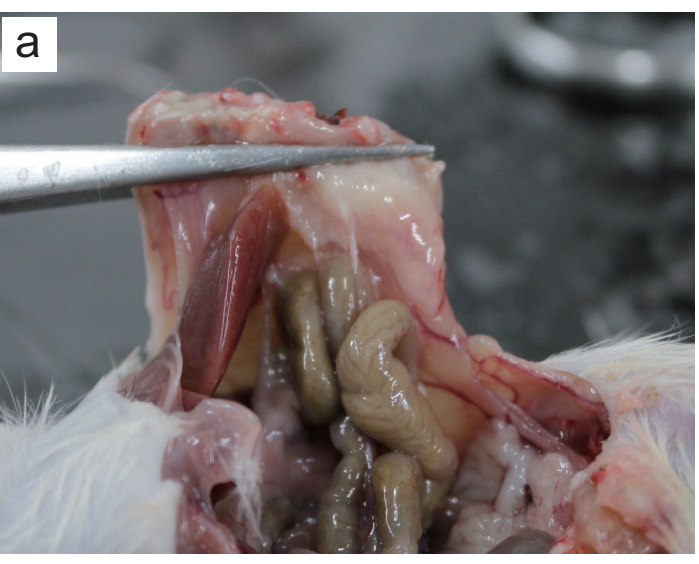

HA-Ph solution: 1 week

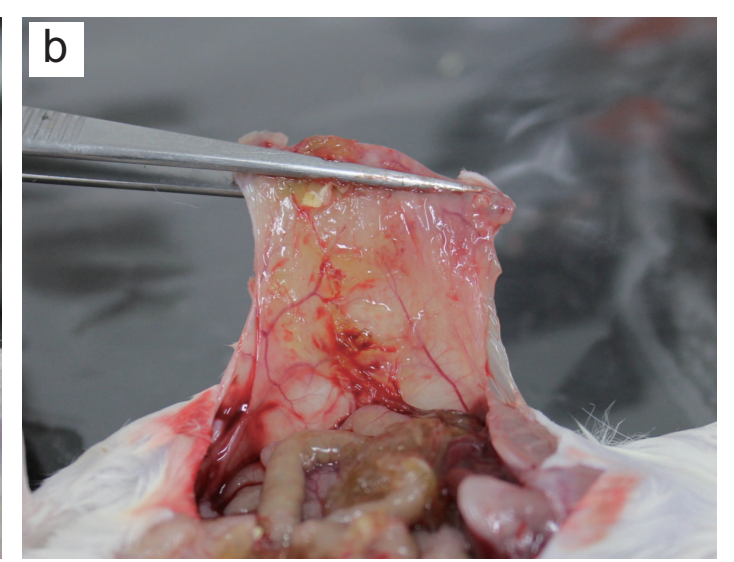

HA-Ph hydrogel: 1 week

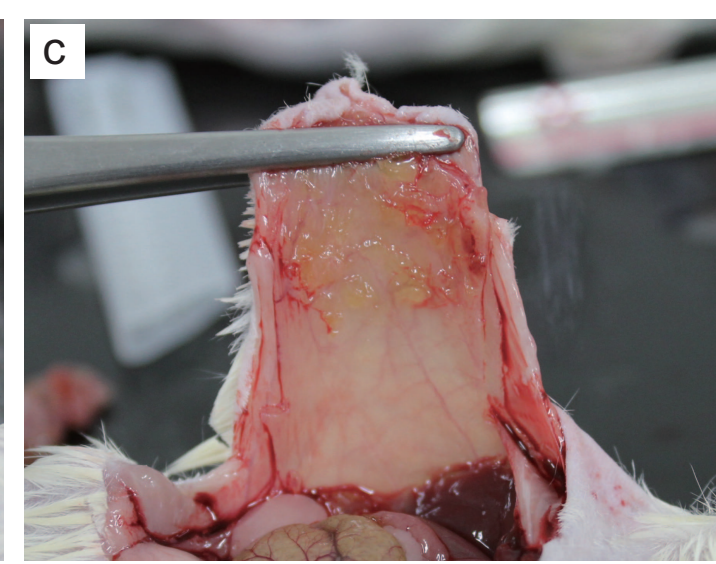

HA-Ph hydrogel: 1 month

Figure 7 
Hyaluronic acid derivative

+ HRP + GOx solution
Body fluid

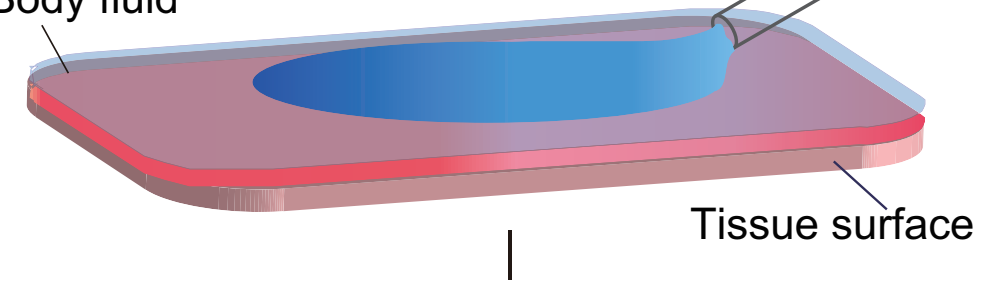

Hydrogelation on tissue surface

$\downarrow$

Peritoneal adhesion prevention

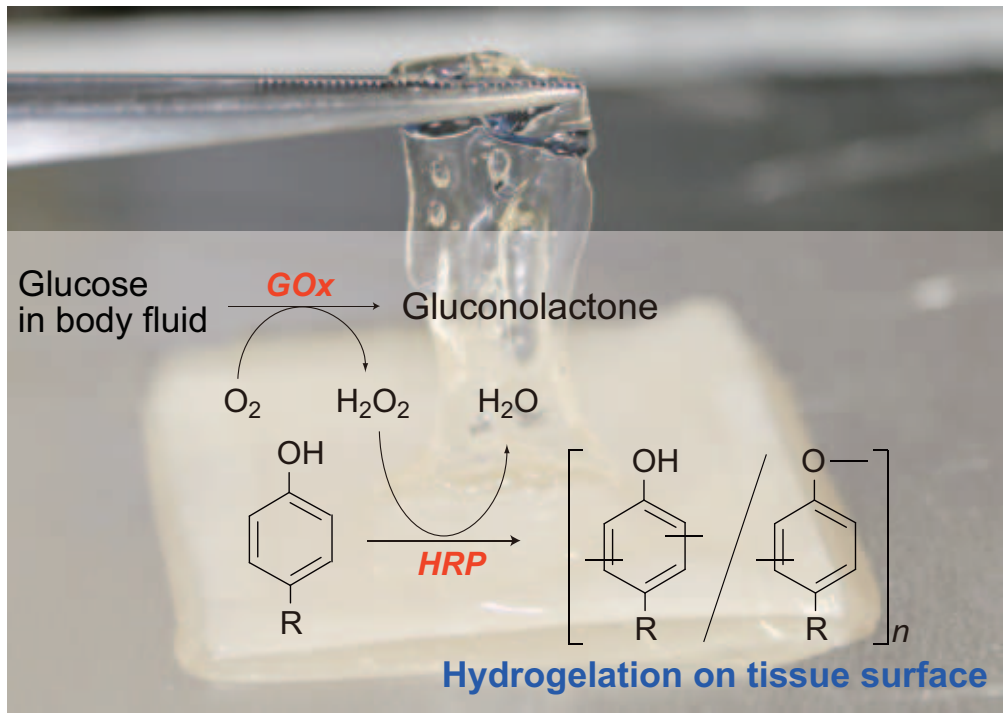

